\title{
Estado de Confiança e Política Econômica Anticíclica em um Modelo Macroeconômico Keynesiano
}

\section{State of Confidence and Anti-cyclical Economic Policy in a Keynesian Macroeconomic Model}

\author{
Guilherme Ricardo dos Santos Souza e Silva* \\ Marcelo Luiz Curado**
}

\begin{abstract}
Resumo: O estado de confiança é um elemento chave para o entendimento do processo decisório dos agentes na teoria keynesiana. Apesar disso, essa variável raramente é encontrada em modelos macroeconômicos formais. A dificuldade em se representar matematicamente uma variável subjetiva e sua dependência das condições psicológicas dos agentes explicam essa ausência. No presente artigo, apresenta-se um modelo macroeconômico formal que incorpora esse elemento e discute a sua relação com a política fiscal executada pelo governo. Ao considerar o impacto do estado de confiança sobre os componentes da demanda agregada, o modelo revela a possibilidade de resultados contraproducentes de uma política fiscal expansionista, resultado que difere de boa parte dos modelos keynesianos tradicionais. O presente trabalho apresenta, portanto, uma crítica às abordagens keynesianas que propõem necessariamente a utilização de políticas expansionistas em períodos de desaceleração econômica.
\end{abstract}

Palavras-chave: Teoria keynesiana. Estado de confiança. Política fiscal. Teoria póskeynesiana.

\begin{abstract}
The state of confidence is a key element for understanding the decision-making process of economic agents in Keynesian theory. Despite this, this variable is rarely found in formal macroeconomic models. The difficulty in representing mathematically a subjective variable and its dependence on the agent's psychological conditions explain this absence. In this paper we present a formal macroeconomic model that incorporates this element and discusses its relationship with the fiscal policy implemented by the government. In considering the impact of the state of confidence on the components of aggregate demand, the model reveals the possibility of counterproductive results of an expansionary fiscal policy, a result that differs from many traditional Keynesian models. The present paper therefore presents a critique of the Keynesian approaches that necessarily propose the use of expansionist policies in periods of economic slowdown.
\end{abstract}

Keywords: Keynesian theory. State of confidence. Fiscal policy. Post-Keynesian theory.

\footnotetext{
* $\quad$ Doutor em Desenvolvimento Econômico pela Universidade Federal do Paraná (UFPR). Professor do Setor de Educação Profissional e Tecnológica da Universidade Federal do Paraná (UFPR). E-mail: guilherme.fdg@uol.com.br

* $\quad$ Doutor em Economia pela Universidade Estadual de Campinas (Unicamp). Professor titular do Departamento de Economia e do Programa de Pós-Graduação em Desenvolvimento Econômico da Universidade Federal do Paraná (UFPR). O autor agradece o financiamento do CNPQ. E-mail: mlcurado@gmail.com
} 
JEL Classification: E12.

\section{Introdução}

Uma das explicações mais difundidas para a recente crise brasileira é que a retração econômica decorreu de um colapso no estado de confiança. Economistas de distintas matrizes teóricas sustentam que essa deterioração reduziu o apetite dos empresários para a realização de gastos com investimentos, comprometendo o potencial de acumulação de capital e de crescimento do sistema.

Ainda que seja um tema recorrente nos debates e análises de conjuntura, pouca atenção tem sido dispensada aos impactos de alterações do estado de confiança sobre a economia em modelos macroeconômicos formais. A literatura keynesiana, especialmente o pós-keynesianismo, é uma exceção a essa regra. Da mesma forma, pode-se afirmar que pouca atenção tem sido dispensada sobre os efeitos de políticas econômicas expansionistas sobre o estado de confiança, mesmo na literatura pós-keynesiana.

Este trabalho tem como objetivo contribuir para reduzir a lacuna entre os modelos teóricos e as discussões que imputam relevância ao papel exercido pela variável estado de confiança na definição do nível de atividade. O artigo incorpora a variável estado de confiança em um modelo macroeconômico keynesiano, através da avaliação do impacto da variável sobre os componentes de demanda.

Além de promover a incorporação da variável, o trabalho também promove uma discussão sobre os determinantes macroeconômicos do estado de confiança. Nesse caso, o trabalho prioriza a relevância dos resultados fiscais na definição do comportamento da variável. A hipótese utilizada é que os resultados fiscais são relevantes na definição do estado de confiança.

O modelo elaborado permite discutir como, sob certas condições, a execução de políticas expansionistas em períodos de crise pode ser contraproducente para promover a recuperação da economia, resultado que contraria boa parte da literatura keynesiana estabelecida. Uma das inovações do trabalho é abordar a ineficiência da política econômica como não resultado - como sugere a macroeconomia convencional - de restrições de oferta ou de qualquer tipo de imperfeição de funcionamento dos mecanismos de mercado. Os limites da política expansionista e a possibilidade de que seja ineficiente decorrem, no modelo, da situação vigente e de suas interações com o estado de confiança.

O artigo está organizado da seguinte forma: após esta breve introdução, a seção 2 apresenta uma discussão sobre o significado do estado de confiança e sua relação com a política econômica e promove uma breve discussão sobre a política econômica em períodos de crise; a seção 3 apresenta os blocos que constituem o modelo macroeconômico de curto prazo; a seção 4 analisa o estado de confiança e seus principais determinantes; a seção 5 desenvolve a solução do modelo; a 
seção 6 discute a influência do déficit fiscal e da dívida pública sobre o estado de confiança; por fim, a seção 7 traça as considerações finais.

\section{Estado de Confiança e Política Econômica}

A discussão sobre o papel do estado de confiança na economia, especialmente na determinação do nível de gastos com investimentos dos empresários, é encontrada originalmente no capítulo 12 da Teoria Geral de Keynes:

The state of confidence, as they term it, is a matter to which practical men always pay the closest and most anxious attention. But economists have not analyzed it carefully and have been content, as a rule, to discuss it in general terms. In particular it has not been made clear that its relevance to economic problems comes in through its important influence on the schedule of the marginal efficiency of capital. There are not two separate factors affecting the rate of investment, namely, the schedule of the marginal efficiency of capital and the state of confidence. The state of confidence is relevant because it is one of the major factors determining the former, which is the same thing as the investment [...]. (KEYNES, 1936, p. 148).

Keynes sustenta, portanto, que o estado de confiança é uma variável chave para as decisões de investimento dos agentes econômicos, tanto do ponto de vista das aplicações financeiras quanto dos investimentos físicos em bens de capital. No entanto, de acordo com o próprio autor, pouco pode-se dizer a priori sobre o que determina o estado de confiança. O autor limita-se a sugerir que a observação dos mercados e da psicologia dos negócios é o melhor caminho para a compreensão do significado e dos impactos do estado de confiança sobre o sistema econômico.

Dois caminhos explicam a importância do estado de confiança na determinação do nível de emprego e renda da economia, de acordo com Keynes (1936). Por um lado, o colapso no estado de confiança reduz a renda esperada dos rendimentos dos bens de capital, reduzindo, portanto, a eficiência marginal do capital. Por outro lado, um colapso no estado de confiança amplia a preferência pela liquidez, o que, para uma dada oferta de moeda, tende a elevar a taxa de juros. Essa combinação de queda na eficiência marginal do capital com ampliação da taxa de juros é a responsável pela queda dos investimentos e da demanda agregada do sistema.

O papel do estado de confiança sobre a economia é tema recorrente na literatura pós-keynesiana contemporânea. Minsky (1982, 1986) e Davidson (1972, 2002), dois dos principais autores que ajudaram na construção e difusão do pós-keynesianismo, dão grande importância ao papel desempenhado pelo estado de confiança na determinação dos rumos do sistema econômico. Minsky (1986), por exemplo, assume que o preço de demanda dos ativos de capital depende, entre outros fatores, do estado de confiança. A melhora no estado de confiança, para 
um dado preço de oferta, eleva a demanda por bens de capital o que, dentro da abordagem desenvolvida pelo autor, implica na tomada de crédito por parte dos empresários e aumento do grau de endividamento das empresas.

Carvalho (2009), em um texto que discute a retomada do interesse sobre as teses originais de Keynes após a crise financeira global de 2008, sumariza a importância da variável estado de confiança para o pensamento keynesiano contemporâneo:

O estado de confiança (e seus conceitos correlatos, como o animal spirits) se torna um elemento essencial de determinação do ponto de demanda efetiva e, assim, um determinante central da produção e do emprego em uma economia empresarial. Um colapso do estado de confiança conduz a um aumento da preferência pela liquidez que, por seu turno, conduz à redução dos preços de demanda dos ativos menos líquidos, atingindo especialmente os ativos de capital real, contraindo investimentos e, através do multiplicador de consumo (ausente nos textos atuais de macroeconomia, que preferiram voltar às hipóteses pré-keynesianas de determinação do consumo pela maximização intertemporal de utilidade), contraindo a renda e o emprego agregados. (CARVALHO, 2009, p. 99).

A importância da variável para os pós-keynesianos não se restringe apenas ao seu papel global na determinação do nível de investimentos, renda e emprego. Harvey (2010), por exemplo, ao sumarizar a literatura pós-keynesiana que busca entender as causas para as frequentes crises nos mercados de ativos, tal como a crise de 2008, atribui papel fundamental ao colapso do estado de confiança para a deflagração dessas crises.

A discussão sobre a variável estado de confiança na literatura econômica convencional é praticamente inexistente. Duas explicações podem ser dadas para a ausência de discussão nos livros textos de macroeconômica: a) de natureza histórica: a popularização dos insights da Teoria Geral, promovida a partir da difusão do modelo IS-LM elaborado por Hicks (1937), deixou de lado, na discussão sobre a determinação do investimento, o papel exercido pela eficiência marginal do capital, o que praticamente implicou ignorar o tema da incerteza e o papel do estado de confiança na economia - em Hicks (1937), o investimento é uma função apenas da taxa de juros; b) de natureza metodológica: a variável estado de confiança não é mensurável, o que limita sua relevância para o novo consenso macroeconômico.

A relação entre a política econômica e o estado de confiança é tema igualmente escasso na literatura convencional. Na literatura de inspiração keynesiana, segue-se a seguinte sequência lógica: uma piora no estado de confiança reduz a renda esperada dos ativos de capital, reduzindo, portanto, a eficiência marginal do capital. Ao mesmo tempo, ao ampliar a preferência pela liquidez, tende-se a observar uma elevação da taxa de juros. O resultado desse processo é a queda 
do investimento, da renda agregada e do emprego. A solução, originalmente proposta por Keynes (1936), é que o governo empreenda políticas anticíclicas como forma de atenuar a queda da demanda agregada.

A indicação do uso de políticas anticíclicas em períodos de queda da demanda, por Keynes (1936), foi popularizada através de sua divulgação no modelo IS-LM elaborado a partir de Hicks (1937). A solução proposta para períodos de redução da atividade era indubitável: promover políticas fiscais, monetárias e creditícias expansionistas. Não é sem motivos que Joan Robinson, no início da década de 1970, batizou essa abordagem keynesiana mecanicista de "keynesianismo bastardo".

Enganam-se, no entanto, aqueles que acreditam que, ao menos no campo da proposição de políticas econômicas em períodos de desaceleração, as indicações keynesianas alteraram-se profundamente. Fritz, Paula e Prates (2016, p. 182), por exemplo, recomendam que:

Quanto à política fiscal, durante a desaceleração econômica, quando as expectativas dos agentes sobre o futuro se deterioram, a política monetária expansionista pode deixar de estimular a demanda agregada devido ao aumento da preferência pela liquidez dos agentes. Sob tais condições, a política monetária deve ser complementada por uma política fiscal expansionista.

Em última análise, Fritz, Paula e Prates (2016), como a maior parte da literatura de inspiração keynesiana, recomendam a execução de políticas expansionistas nos campos monetário, creditício e fiscal em períodos de desaceleração econômica.

As críticas da literatura convencional à execução de políticas expansionistas em contextos de crise são amplamente conhecidas. Milton Friedman e os monetaristas, assim como Robert Lucas e a macroeconomia novo-clássica estabeleceram críticas contundentes sobre a eficiência das políticas econômicas anticíclicas baseadas na capacidade dos agentes econômicos que formam expectativas de forma adaptativa ou racional de antecipar os efeitos da política sobre a inflação, o que elimina em termos reais os efeitos da política econômica expansionista, tornando-a ineficiente.

As críticas elaboradas por monetaristas e novo-clássicos, ainda que fundamentais na construção da macroeconomia moderna, passaram à margem dos debates pós-keynesianos. Ao mesmo tempo, no campo da ação concreta dos gestores de política, períodos de crise ensejaram a adoção de políticas expansionistas com resultados bastante diversos. Esses dois fatores combinados parecem ser relevantes para entender porque até hoje as indicações de política econômica para recuperação da economia na literatura keynesiana mantêm praticamente inalterada as sugestões originalmente propostas por Keynes (1936). 
A partir da próxima seção inicia-se a construção dos blocos fundamentais do modelo macroeconômico que incorpora a variável estado de confiança e estabelece suas relações com a política econômica, especialmente a política fiscal. Como ficará claro, as relações existentes entre a política fiscal e o estado de confiança podem reduzir a capacidade da primeira em promover a recuperação. Se a realização de uma política fiscal expansionista promover a piora do estado de confiança, os efeitos multiplicadores tradicionais podem ser neutralizados, tornando a política ineficiente. Os limites da política expansionistas independem no modelo da antecipação do comportamento da inflação ou de restrições de oferta, típicas de modelos convencionais.

\section{Modelo Macroeconômico}

Esta seção apresenta os elementos que constituem o modelo macroeconômico keynesiano para uma economia fechada. ${ }^{1}$

\section{I Função de Produção e Mercado de Trabalho}

O modelo utiliza uma função de produção padrão em que o produto é uma função do estoque de capital e da quantidade de trabalho empregada, representada pela equação:

$$
\mathrm{Y}=\mathrm{F}(\mathrm{K}, \mathrm{N})
$$

Assume-se também que o salário real é igual à produtividade marginal do trabalho, portanto:

$$
\mathrm{w} / \mathrm{p}=\mathrm{F}_{\mathrm{N}}
$$

Como se trata de um modelo keynesiano de curto prazo, nenhuma suposição é feita a respeito do equilíbrio no mercado de trabalho.

\subsection{Ativos Financeiros}

Os agentes econômicos podem compor sua riqueza em dois ativos: moeda e títulos públicos. A posse da moeda é justificada exclusivamente por seu atributo da liquidez, e não gera evidentemente nenhum ganho monetário. A posse dos títulos permite ao agente o recebimento de juros (cujo valor depende da taxa de juros

1 Entre as diversas referências que embasaram os blocos que compõem o modelo apresentado, citam-se Keynes (1936), Meade (1937), Tobin (1969), Sargent (1987) e Blanchard e Fischer (1989). 
r). A demanda dos agentes por moeda, além de depender diretamente do nível de renda e inversamente da taxa de juros, conforme a maior parte dos modelos keynesianos, depende também, no presente modelo, do estado de confiança dos agentes.

Quanto mais elevado o estado de confiança, maior é a crença dos agentes de que suas previsões em relação ao futuro se concretizarão. Por uma questão de simplicidade, considera-se que quando o estado de confiança estiver elevado, os agentes também estarão otimistas em relação ao futuro da economia, visto que em uma situação de grande incerteza, na grande maioria dos casos, há uma tendência dominante do pessimismo entre os agentes.

Assim, seguindo a discussão keynesiana a respeito da preferência pela liquidez, um estado de confiança deteriorado ou uma situação de grande incerteza em relação ao futuro da economia amplia a demanda por moeda, o ativo mais líquido disponível para os agentes econômicos manterem sua riqueza.

\subsection{Componentes de Demanda}

Seguindo a tradição keynesiana, assume-se que o consumo dos agentes dependerá da renda disponível $(Y-T)$. A taxa de juros também influencia o nível de consumo dos agentes, uma vez que uma elevação na taxa de juros torna o consumo presente mais caro em relação ao consumo futuro, induzindo os consumidores a aumentarem o seu nível de poupança. O terceiro elemento que determina o nível de consumo dos agentes é o seu estoque de riqueza. Como no modelo tem-se apenas dois ativos, moeda e títulos, a soma do estoque real de riqueza terá impacto sobre o nível de consumo.

Um quarto elemento importante que influencia o consumo dos agentes e que está bastante associado com os desenvolvimentos da linha pós-keynesiana é o estado de confiança. Assim, o consumo também é influenciado pela variável $\varphi$, que representa o estado de confiança dos agentes. Quanto maior o valor de $\varphi$, maior é a confiança dos agentes em suas previsões sobre os eventos futuros. O consumo é influenciado pelo estado de confiança porque, em uma situação de elevada incerteza, os agentes tendem a reduzir o seu consumo, tanto os trabalhadores pelo receio em relação à possibilidade de desemprego, quanto os empresários pela perspectiva de redução nos lucros futuros. Assim:

$$
C=C\left(Y-T, r, \frac{M+B}{p}, \varphi\right)
$$

A função investimento, representada pela equação 4, informa que o investimento depende do nível de produto, da taxa de juros e da variável psicológica . No caso de inversões em bens de capital, conforme destacado por Keynes (1936) 
na Teoria Geral, o impacto do estado de confiança sobre as decisões de investimento é ainda maior. De fato, a decisão de investimento será tomada com base na expectativa de lucros futuros decorrentes da posse e utilização dos bens de capital pelas empresas. Assim:

$$
\mathrm{I}=\mathrm{I}(\mathrm{Y}, \mathrm{r}, \varphi)
$$

A equação 5 apresenta a composição da demanda agregada para a economia fechada e representa a condição de equilíbrio no mercado de bens e serviços, ou seja, a condição de igualdade entre oferta e demanda, ${ }^{2}$ representando a curva IS do sistema.

$$
\text { IS: } \mathrm{Y}=\mathrm{C}+\mathrm{I}+\mathrm{G}
$$

\subsection{Mercado de Ativos: Moeda e Títulos}

O equilíbrio no mercado financeiro exige que os dois mercados (moeda e títulos públicos) estejam em equilíbrio. A restrição de portfólio é baseada no trabalho de Tobin (1969). As equações 6 e 7 apresentam as funções de demanda por moeda e títulos públicos. Conforme discutido anteriormente, a demanda de moeda é influenciada pela taxa de juros (inversamente), pela renda (diretamente pelo motivo de transação) e pelo estado de confiança $\varphi$, visto que, em uma situação de elevada incerteza, a demanda por moeda aumenta. Estão implícitas nas equações as condições de equilíbrio de cada mercado, quando a demanda pelo ativo é igual à sua oferta.

$$
\begin{aligned}
& m(r, Y, \varphi)=\frac{M}{p} \\
& b(r, Y, \varphi)=\frac{B}{p}
\end{aligned}
$$

A equação 8 é a restrição de portfólio, em que a riqueza real dos agentes é igual à soma do estoque real de moeda e títulos públicos.

$$
W=\frac{M}{p}+\frac{B}{p}
$$

Diferenciando-se totalmente as equações 1 a 5, tem-se:

$$
d Y=F_{K} d K+F_{N} d N
$$

$\overline{2}$ O modelo assume que os gastos do governo $(G)$ são exógenos. 


$$
\begin{gathered}
\frac{d w}{w}-\frac{d p}{p}=\frac{F_{N N}}{F_{N}} d N \\
d C=C_{1} d Y-C_{1} d T+C_{2} d r+C_{3}\left[\frac{d M+d B}{p}-\left(\frac{d w}{w}-\frac{F_{N N}}{F_{N}^{2}} d Y\right) \frac{M+B}{p}\right]+C_{4} d \varphi \\
\mathrm{dI}=\mathrm{I}_{1} \mathrm{dY}+\mathrm{I}_{2} \mathrm{dr}+\mathrm{I}_{3} \mathrm{~d} \varphi \\
\mathrm{d} \mathrm{Y}=\mathrm{dC}+\mathrm{dI}+\mathrm{dG}
\end{gathered}
$$

\section{O Estado de Confiança e a Política Econômica}

Até o momento, considerou-se que o nível de consumo dos agentes, o nível de investimento privado e a demanda por moeda são funções da variável psicológica , que representa o estado de confiança dos agentes econômicos. É preciso deixar claro que não é objetivo desta seção uma discussão mais ampla sobre os determinantes do estado de confiança. O objetivo é bem menos pretencioso. Pretende-se apenas analisar a relação entre o estado de confiança e a política econômica, especialmente a política fiscal.

A hipótese utilizada é que as condições fiscais exercem um papel relevante na definição do estado de confiança. Resultados fiscais frágeis ou considerados insustentáveis no tempo deterioram as expectativas dos agentes quanto ao futuro da economia. Situações fiscais graves podem levar, no limite, ao surgimento de expectativas de default, ou, em casos menos graves, a duros ajustes fiscais que via de regra reduzem o nível de atividade econômica e as expectativas de lucros futuros dos empresários. Objetivamente, utilizam-se como determinantes fiscais do estado de confiança o déficit público elevado, especialmente quando associado a um alto estoque de endividamento público e ao crescimento da relação entre dívida pública e produto. Essas variáveis são relevantes para explicar um processo de deterioração do estado de confiança, especialmente em países com histórico de fragilidade nas contas públicas.

Evidentemente, uma série de outras variáveis exógenas a esse modelo, como aquelas associadas ao desempenho do setor externo da economia e à qualidade das instituições do país, afetam o estado de confiança. Todas essas variáveis exógenas serão denotadas por .

A partir das considerações, define-se que é uma função do déficit público , e do nível de endividamento público $B$. As demais variáveis exógenas estão agrupadas na variável. Assim:

$$
\varphi=\varphi(\mathrm{T}-\mathrm{G}, \mathrm{B}, \Psi)
$$


de forma que:

$$
d \varphi=\varphi_{1} d(T-G)+\varphi_{2} d B+\varphi_{3} d \Psi
$$

\section{A Solução do Modelo}

Para se determinar a solução do sistema, utiliza-se um aparato similar ao utilizado por Hicks (1937). O locus da curva IS é dado pela combinação de taxa de juros e produto que satisfazem a equação 5. Substituindo-se as equações 9, 10, 11, 12 e 15 na equação $13, \operatorname{com} d K=0$ (pois trata-se de um modelo de curto prazo), tem-se:

$$
\begin{aligned}
& d Y=C_{1} d Y-C_{1} d T+C_{2} d r+C_{3}\left[\frac{d M+d B}{p}-\left(\frac{d w}{w}-\frac{F_{N N}}{F_{N}^{2}} d Y\right) \frac{M+B}{p}\right]+C_{4}\left[\varphi_{1} d(T-G)+\right. \\
& \left.\varphi_{2} d B+\varphi_{3} d \Psi\right]+I_{1} d Y+I_{2} d r+I_{3}\left[\varphi_{1} d(T-G)+\varphi_{2} d B+\varphi_{3} d \Psi\right]+d G
\end{aligned}
$$

Reorganizando, tem-se:

$$
\begin{aligned}
& {\left[1-C_{1}-I_{1}-\left(\frac{C_{3}}{p}\right) \frac{F_{N N}}{F_{N}^{2}}(M+B)\right] d Y=\left(C_{4} \varphi_{1}+I_{3} \varphi_{1}-C_{1}\right) d T+\left(1-C_{4} \varphi_{1}+I_{3} \varphi_{1}\right) d G+} \\
& \left(C_{2}+I_{2}\right) d r+\left(\frac{C_{3}}{p}\right) d M+\left(\frac{C_{3}}{p}+C_{4} \varphi_{2}+I_{3} \varphi_{2}\right) d B-\frac{C_{3}(M+B)}{p} \frac{d w}{w}+\left[C_{4}+I_{3}\right] \varphi_{3} d \Psi
\end{aligned}
$$

A inclinação da curva IS é, então, dada por:

$$
\left.\frac{d r}{d Y}\right|_{I S}=\frac{1-C_{1}-I_{1}-\left(\frac{C_{3}}{p}\right)\left[\frac{F_{N N}}{F_{N}^{2}}(M+B)\right]}{C_{2}+I_{2}}
$$

O denominador do lado direito da equação é negativo, pois . O numerador pode ser de ambos os sinais, e se

$$
\left[1-C_{1}-I_{1}-\left(\frac{C_{3}}{p}\right)\left(\frac{F_{N N}}{F_{N}^{2}}(M+B)\right)\right]>0
$$

a curva IS é negativamente inclinada (condição padrão).

O locus da curva LM é obtido pelas combinações de taxa de juros e produto que garantem o equilíbrio nos mercados financeiros (moeda e títulos). Pela lei de Walras, se um dos mercados estiver em equilíbrio o outro também necessariamente estará. Considerando-se o equilíbrio no mercado monetário, tem-se:

$$
\mathrm{LM}: \mathrm{m}(\mathrm{r}, \mathrm{Y}, \varphi)=\mathrm{M} / \mathrm{p}
$$

A equação 20 representa o locus de equilíbrio nos mercados financeiros. Tomando-se o diferencial total da equação 20, tem-se: 


$$
d[m(r, Y, \varphi)]=d\left(\frac{M}{p}\right)
$$

Considerando-se que

$$
d[m(r, Y, \varphi)]=m_{r} d r+m_{Y} d Y+m_{\varphi} d \varphi
$$

tem-se:

$$
m_{r} d r+m_{Y} d Y+m_{\varphi} d \varphi=\frac{d M}{p}-\frac{M}{p}\left(\frac{d w}{w}-\frac{F_{N N}}{F_{N}^{2}} d Y\right)
$$

Substituindo-se a equação 15 na equação 23, tem-se:

$$
m_{r} d r+m_{Y} d Y+m_{\varphi}\left[\varphi_{1} d(T-G)+\varphi_{2} d B+\varphi_{3} d \Psi\right]=\frac{d M}{p}-\frac{M}{p}\left(\frac{d w}{w}-\frac{F_{N N}}{F_{N}^{2}} d Y\right)
$$

Assim, a inclinação da curva LM é dada por:

$$
\left.\frac{d r}{d Y}\right|_{L M}=\frac{\left(\frac{M}{p}\right) \frac{F_{N N}}{F_{N}^{2}}-m_{Y}}{m_{r}}
$$

A curva LM é positivamente inclinada, pois:

$$
m_{r}<0, m_{Y}>0 e \frac{F_{N N}}{F_{N}^{2}}<0
$$

O ponto de equilíbrio das curvas IS e LM pode ser obtido analiticamente, substituindo-se $d r$ na equação (17):

$$
\begin{aligned}
& \left\{1-C_{1}-I_{1}-\left(\frac{C_{3}}{p}\right) \frac{F_{N N}}{F_{N}^{2}}(M+B)-\frac{\left(C_{2}+I_{2}\right)}{m_{r}}\left[\left(\frac{M}{p}\right) \frac{F_{N N}}{F_{N}^{2}}-m_{Y}\right]\right\} d Y=-C_{1} d T+d G+ \\
& \frac{\left(C_{2}+I_{2}\right)\left(1+C_{3}\right)}{m_{r}}\left(\frac{d M}{p}\right)+\left(\frac{C_{3}}{p}\right) d B-\left[\frac{\left(C_{2}+I_{2}\right)}{m_{r}} \frac{M}{p}+\frac{C_{3}(M+B)}{p}\right] \frac{d w}{w}+\left[\left(C_{4}+I_{3}-\frac{\left(C_{2}+I_{2}\right)}{m_{r}} m_{\varphi}\right)\right]\left[\varphi_{1} d(T-\right. \\
& \left.G)+\varphi_{2} d B+\varphi_{3} d \Psi^{\prime}\right]
\end{aligned}
$$

Denominando-se $H=1-C_{1}-I_{1}-\left(\frac{C_{3}}{p}\right) \frac{F_{N N}}{F_{N}^{2}}(M+B)-\frac{\left(C_{2}+I_{2}\right)}{m_{r}}\left[\left(\frac{M}{p}\right) \frac{F_{N N}}{F_{N}^{2}}-m_{Y}\right]$ e reorganizando-a, tem-se:

$$
\begin{aligned}
& H d Y=\left[\left(C_{4}+I_{3}-\frac{\left(C_{2}+I_{2}\right)}{m_{r}} m_{\varphi}\right) \varphi_{1}-C_{1}\right] d T+\left[1-\left(C_{4}+I_{3}-\frac{\left(C_{2}+I_{2}\right)}{m_{r}} m_{\varphi}\right) \varphi_{1}\right] d G+ \\
& \frac{\left(C_{2}+I_{2}\right)\left(1+C_{3}\right)}{m_{r}}\left(\frac{d M}{p}\right)+\left(\frac{C_{3}}{p}+\left(C_{4}+I_{3}-\frac{\left(C_{2}+I_{2}\right)}{m_{r}} m_{\varphi}\right)\right) \varphi_{2} d B-\left[\frac{\left(C_{2}+I_{2}\right)}{m_{r}} \frac{M}{p}+\frac{C_{3}(M+B)}{p}\right] \frac{d w}{w}+ \\
& {\left[\left(C_{4}+I_{3}-\frac{\left(C_{2}+I_{2}\right)}{m_{r}} m_{\varphi}\right)\right] \varphi_{3} d \Psi}
\end{aligned}
$$




\subsection{Análise de Estabilidade do Modelo}

A estabilidade do sistema é verificada de modo padrão. Se a demanda agregada é maior do que a oferta agregada, os preços tendem a subir. Se há um desequilíbrio nos mercados financeiros, as taxas de juros tendem a mudar de modo a equilibrá-los. No modelo, alterações no produto seguem o nível de preços, enquanto o sistema se move ao longo da curva de oferta agregada:

$$
\mathrm{p}=\mathrm{p}(\mathrm{Y}, \mathrm{w}, \mathrm{K})
$$

A função que relaciona o nível de preços ao produto é dada pelas equações 9 e 10:

$$
\frac{d p}{p}=-\frac{F_{N N}}{F_{N}^{2}} d Y
$$

ou

$$
d Y=-\frac{w}{p^{2}} \frac{F_{N}}{F_{N N}} d p
$$

A seguir, é necessário definir as equações diferenciais que representam a trajetória temporal dos preços e das taxas de juros:

$$
\begin{gathered}
\frac{d p}{d t}=\alpha\left[C\left(Y-T, r, \frac{M+B}{p}\right)+I(Y, r, \varphi)+G-Y\right] \\
\alpha^{\prime}>0 \text { e } \alpha(0)=0 \\
\frac{d r}{d t}=\beta\left(m(r, Y, \varphi)-\frac{M}{p}\right) \\
\beta^{\prime}>0 e \beta(0)=0
\end{gathered}
$$

$\alpha^{\prime}$ é positivo porque, se a demanda agregada por bens e serviços for maior do que a oferta agregada, os preços tendem a subir. é positivo porque, se a demanda por moeda excede a oferta de moeda, as taxas de juros tendem a subir. Tomando-se uma aproximação linear dessas equações ao redor dos valores de equilíbrio, tem-se:

$$
\begin{gathered}
\frac{d p}{d t}=\alpha^{\prime}\left[1-C_{1}-I_{1}-\left(\frac{C_{3}}{p}\right)\left(\frac{F_{N N}}{F_{N}^{2}} \frac{M+B}{p}\right)\right] \frac{w}{p^{2}} \frac{F_{N}}{F_{N N}}\left(p-p_{0}\right)+\alpha^{\prime}\left(C_{2}+I_{2}\right)\left(r-r_{0}\right) \\
\frac{d r}{d t}=-\beta^{\prime}\left[\left(\frac{M}{p}\right) \frac{F_{N N}}{F_{N}^{2}}-m_{Y}\right]\left(p-p_{0}\right)+\beta^{\prime}\left[m_{r}\right]\left(r-r_{0}\right)
\end{gathered}
$$

Observa-se que (34) e (35) são equações diferenciais de primeira ordem, cuja equação característica é dada por: 


$$
\left|\begin{array}{cc}
a-\lambda & b \\
c & d-\lambda
\end{array}\right|=\lambda^{2}-(a+d) \lambda+(a d-b c)=0
$$

em que:

$$
\begin{aligned}
& a=\alpha^{\prime}\left[1-C_{1}-I_{1}-\left(\frac{C_{3}}{p}\right)\left(\frac{F_{N N}}{F_{N}^{2}} \frac{M+B}{p}\right)\right] \frac{w}{p^{2}} \frac{F_{N}}{F_{N N}} \\
& b=\alpha^{\prime}\left(C_{2}+I_{2}\right) \\
& c=-\beta^{\prime}\left[\left(\frac{M}{p}\right) \frac{F_{N N}}{F_{N}^{2}}-m_{Y}\right] \\
& d=\beta^{\prime}\left[m_{r}\right]
\end{aligned}
$$

As condições necessárias e suficientes para que o sistema seja estável são: $-(a+d)>0$ e $(a d-b c)>0$. Dessa forma, a primeira condição pode ser escrita como:

$$
-\left\{\alpha^{\prime}\left[1-C_{1}-I_{1}-\left(\frac{C_{3}}{p}\right)\left(\frac{F_{N N}}{F_{N}^{2}} \frac{M+B}{p}\right)\right] \frac{w}{p^{2}} \frac{F_{N}}{F_{N N}}+\beta^{\prime}\left[m_{r}\right]\right\}>0
$$

e a segunda condição como:

$$
\left\{\alpha^{\prime}\left[1-C_{1}-I_{1}-\left(\frac{C_{3}}{p}\right)\left(\frac{F_{N N}}{F_{N}^{2}} \frac{M+B}{p}\right)\right] \frac{w}{p^{2}} \frac{F_{N}}{F_{N N}}\right\} \beta^{\prime}\left[m_{r}\right]+\alpha^{\prime} \quad\left(\quad C_{2}+I_{2}\right) \beta^{\prime}\left[\left(\frac{M}{p}\right) \frac{F_{N N}}{F_{N}^{2}}-m_{Y}\right]>0
$$

Reorganizando, tem-se:

$$
\frac{1-C_{1}-I_{1}-\left(\frac{C_{3}}{p}\right)\left[\frac{F_{N N}}{F_{N}^{2}}(M+B)\right]}{C_{2}+I_{2}}>-\frac{\left(\frac{M}{p}\right) \frac{F_{N N}}{F_{N}^{2}}-m_{Y}}{m_{r}}
$$

que é equivalente a:

$$
\left.\frac{\partial r}{\partial Y}\right|_{I S}-\left.\frac{\partial r}{\partial Y}\right|_{L M}<0
$$

Portanto, uma condição necessária para a estabilidade do sistema é que a curva LM seja mais inclinada do que a curva IS. Naturalmente, se a curva LM for positivamente inclinada e a curva IS negativamente inclinada (situação padrão), a condição é verificada. Mesmo se a curva IS for positivamente inclinada, é possível que a segunda condição se verifique, desde que a inclinação da curva LM seja maior do que da curva IS.

Como $\alpha^{\prime}$ e $\beta$ ' são ambos positivos $\left(e \mathrm{~F}_{\mathrm{N}}>0, \mathrm{~F}_{\mathrm{NN}}<0\right)$, a primeira condição é satisfeita se:

$$
\left[1-C_{1}-I_{1}-\left(\frac{C_{3}}{p}\right)\left(\frac{F_{N N}}{F_{N}^{2}} \frac{M+B}{p}\right)\right] \frac{w}{p^{2}} \frac{F_{N}}{F_{N N}}>0 ;\left[m_{r}\right]<0
$$

A primeira condição é verificada se a curva IS é negativamente inclinada. A segunda condição é verificada sempre, já que $\left[m_{r}\right]<0$. No entanto, mesmo se 
a curva IS for positivamente inclinada, a condição pode ser verificada, mas isso dependerá das magnitudes relativas das velocidades de ajustamento $\alpha^{\prime}$ e $\beta$ ', de acordo com o Gráfico 1.

\section{Gráfico 1 - Sistemas estáveis}
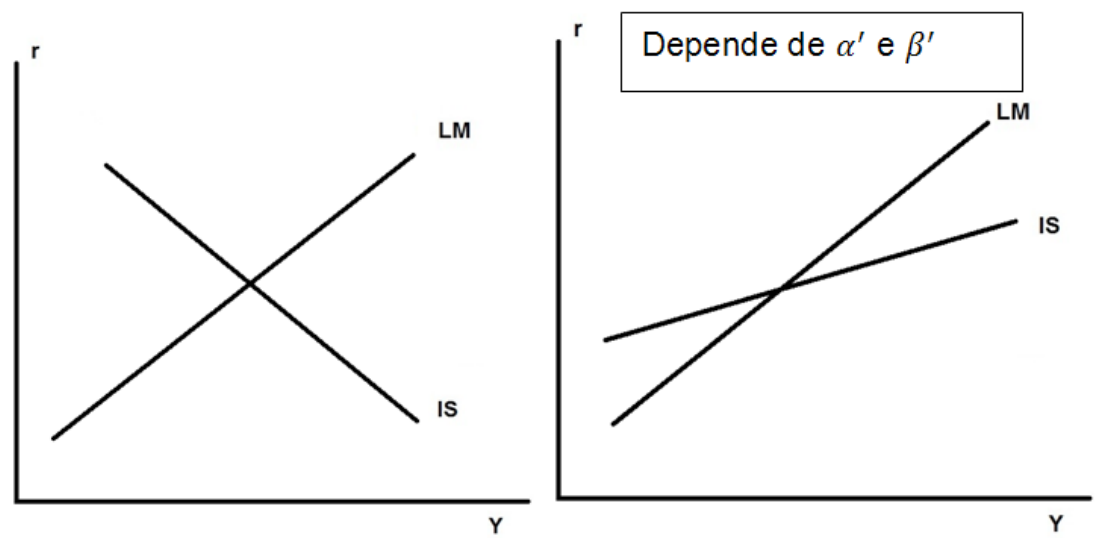

Fonte:Elaborado pelos autores

Resumindo, as condições para estabilidade são:

a) LM mais inclinada do que a IS (condição necessária);

b) curva IS negativamente inclinada;

c) curva LM positivamente inclinada.

As condições $b$ e $c$, em conjunto, são condições suficientes. Contudo, o sistema ainda pode ser estável mesmo que a IS seja positivamente inclinada, mas a seguinte expressão deve ser verificada:

$$
\alpha^{\prime}\left[1-C_{1}-I_{1}-\left(\frac{C_{3}+I_{3}}{p}\right)\left(\frac{F_{N N}}{F_{N}^{2}} \frac{V}{p}+V_{Y}\right)\right] \frac{w}{p^{2}} \frac{F_{N}}{F_{N N}}+\beta^{\prime}\left[(M+V) m_{r}+M b_{r}+\left(\frac{M}{M+B+V}\right) V_{r}\right]<0
$$

Nesse caso as velocidades de ajustamento $\alpha^{\prime}$ e $\beta^{\prime}$ devem ser conhecidas. Não obstante, a curva LM deve ter uma inclinação maior do que a curva IS (uma condição necessária).

\section{Estado de Confiança e Resultado Fiscal}

A partir da solução analítica do modelo pode-se verificar os resultados da economia em termos de estática comparativa. Se a curva IS é negativamente inclinada (condição padrão), $\mathrm{H}>0$, tem-se: 


$$
\begin{gathered}
\frac{\partial Y}{\partial G}=\frac{\left[1-\left(C_{4}+I_{3}-\frac{\left(C_{2}+I_{2}\right)}{m_{r}} m_{\varphi}\right) \varphi_{1}\right]}{H}=? \\
\frac{\partial Y}{\partial T}=\frac{\left[\left(C_{4}+I_{3}-\frac{\left(C_{2}+I_{2}\right)}{m_{r}} m_{\varphi}\right) \varphi_{1}-C_{1}\right]}{H}=? \\
\frac{\partial Y}{\partial M}=\frac{\frac{\left(C_{2}+I_{2}\right)\left(1+C_{3}\right)}{m_{r}}}{H}>0 \\
\frac{\partial Y}{\partial B}=\frac{\left(\frac{C_{3}}{p}+\left(C_{4}+I_{3}-\frac{\left(C_{2}+I_{2}\right)}{m_{r}} m_{\varphi}\right)\right) \varphi_{2}}{H}<0
\end{gathered}
$$

Dessa forma, uma política fiscal expansionista (um aumento nos gastos públicos, por exemplo) pode ter impactos consideravelmente distintos sobre o sistema econômico, a depender do efeito do déficit público sobre o estado de confiança $\left(\varphi_{1}\right)$. Ampliações nos gastos públicos, sob determinadas condições, podem deteriorar o estado de confiança, conforme a equação 42. A explicação para esse resultado é bastante intuitiva. Realizar uma expansão fiscal em uma economia que apresenta significativo superávit primário e possui uma relação dívida/PIB baixa para seu grau de desenvolvimento tem um impacto distinto da realização da mesma política para uma economia com desequilíbrio fiscal crônico e alto grau de endividamento.

Assim, a resposta do investimento a quedas no estado de confiança $\left(\mathrm{I}_{3}\right)$, o impacto do estado de confiança sobre o consumo $\left(\mathrm{C}_{4}\right)$ e a influência da queda no estado de confiança sobre a demanda de moeda $\left(\mathrm{m}_{\varphi}\right)$ determinarão, em conjunto com os parâmetros que elevam o consumo e o investimento devido a uma expansão nos gastos, o efeito líquido de uma política fiscal expansionista.

De acordo com a equação 42, a condição para que uma política fiscal expansionista (um aumento em $G$ ) acarrete uma ampliação no nível de produto é:

$$
\left(C_{4}+I_{3}-\frac{\left(C_{2}+I_{2}\right)}{m_{r}} m_{\varphi}\right) \varphi_{1}<1
$$

O resultado do lado esquerdo da inequação 46 é necessariamente um número positivo, podendo assumir valores maiores ou menores que 1 . Dessa forma, a condição limite para que a política fiscal seja eficiente é dada por:

$$
C_{4}+I_{3}<\frac{1}{\varphi_{1}}+\frac{\left(C_{2}+I_{2}\right)}{m_{r}} m_{\varphi}
$$

A interpretação da condição da expressão 47 é bastante intuitiva. A política fiscal expansionista terá resultados positivos: 
a) quanto menos o consumo e o investimento forem sensíveis às variações no estado de confiança dos agentes;

b) quanto menos o estado de confiança for afetado pelo superávit/déficit público (T-G);

c) quanto mais a taxa de juros influenciar a demanda por moeda;

d) quanto menos o consumo e o investimento forem sensíveis às alterações na taxa de juros;

e) quanto menos o estado de confiança influenciar a demanda por moeda.

O Gráfico 2 apresenta a resposta de uma política fiscal expansionista em função da sensibilidade do estado de confiança às alterações no superávit/déficit público. A constante $K$ representa o conjunto das variáveis influenciadas pela taxa de juros .

Figura 2 - Política Fiscal e Estado de Confiança

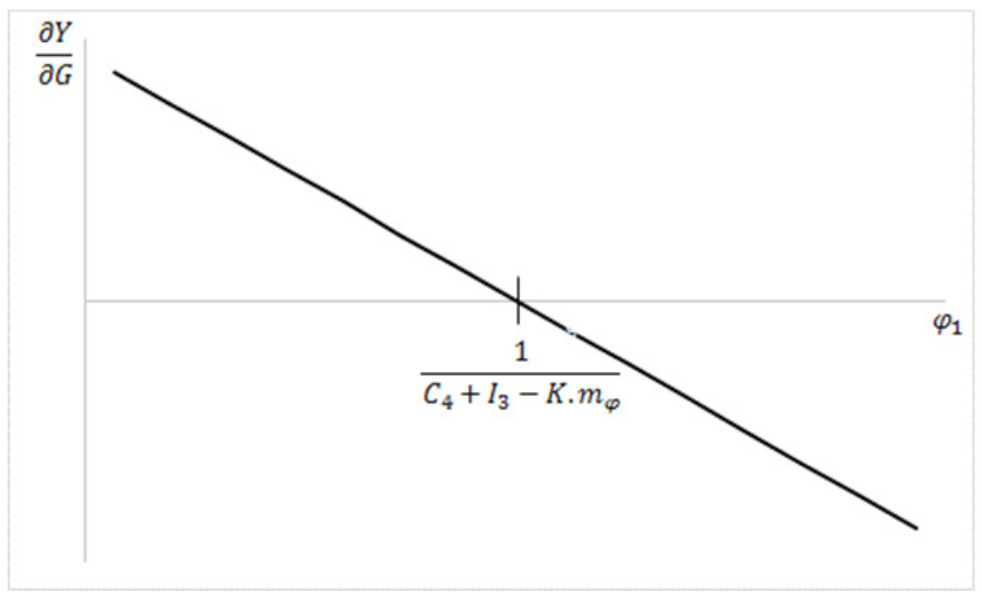

Fonte: Elaborado pelos autores

Em outras palavras, a política fiscal expansionista será efetiva sempre que , o que é equivalente à condição da expressão 47.

Alterações no nível de tributação da renda $(T)$ também podem ter diferentes impactos sobre o produto agregado da economia, pelos mesmos canais discutidos anteriormente para uma expansão dos gastos públicos, conforme mostra a equação 43. O efeito líquido de um aumento da tributação dependerá da magnitude dos parâmetros, conforme mencionado no parágrafo anterior.

Se o estado de confiança for deteriorado por ampliações no estoque de dívida pública $\left(\varphi_{2}\right)$, questão que deve ser analisada em conjunto com o déficit fiscal, há uma tendência de queda no nível de produto a partir de ampliações no estoque 
da dívida. Essa avaliação pode ser feita a partir da equação 45. Uma ampliação do estoque da dívida em conjunto com uma elevação no déficit público tende a ter um considerável impacto sobre o estado de confiança, gerando, a partir das equações 42 e 45 , relevante contração no produto a partir de uma política fiscal expansionista.

\section{Considerações Finais}

O presente trabalho desenvolveu um modelo macroeconômico keynesiano para uma economia fechada. O modelo incorpora formalmente a variável estado de confiança como determinante do comportamento da economia. O trabalho apresenta dois resultados centrais. O primeiro, comum na literatura keynesiana, é que o estado de confiança é um elemento relevante para a determinação do nível de atividade da economia. Nesse caso, a contribuição do trabalho encontra-se em apresentar a variável em um modelo macroeconômico formal simplificado.

O segundo resultado gerado pelo modelo é que a eficiência da política fiscal irá depender de seu impacto sobre o estado de confiança. No modelo, é possível que a ampliação dos gastos públicos deteriore o estado de confiança dos agentes. Nesse caso, políticas expansionistas, que via de regra geram expansão do produto em modelos keynesianos, podem ser contraproducentes, gerando inclusive retração da atividade. Isso poderá ocorrer quando a deterioração do estado de confiança reduzir consumo, investimento e ampliar a demanda por moeda, levando, portanto, a uma contração da demanda agregada. Assim, sem levar em consideração restrições de oferta típicas de modelos macroeconômicos convencionais, é possível que a execução de políticas fiscais expansionistas gerem retração do produto, caso atuem no sentido de deteriorar o estado de confiança da economia.

Dessa forma, pode-se afirmar que o modelo elaborado apresenta uma crítica não convencional à recomendação de política econômica comum em diversas correntes keynesianas. A recomendação de política tipicamente keynesiana funciona como uma receita de bolo: a desaceleração da economia deve ser necessariamente compensada pela adoção de políticas expansionistas, independentemente das condições vigentes no sistema econômico. Recomenda-se a redução de taxas de juros e o aumento de gastos públicos independentemente das condições vigentes no mercado de crédito ou dos resultados fiscais vigentes.

O resultado do modelo deste artigo é que a indicação de uma política fiscal expansionista em um cenário de desaceleração deve levar em conta seus impactos sobre o estado de confiança. Um exemplo recente da economia brasileira pode ajudar na compreensão desses resultados: a incapacidade das políticas fiscais expansionistas promovidas pelo governo Dilma para estimular a economia entre 2011 e 2014 podem ser explicadas como resultado de uma deterioração do estado 
de confiança, dada a fragilidade fiscal que foi sendo gerada pela adoção dessas políticas. Vale lembrar que, entre 2011 e 2014, os resultados fiscais apresentados pelo governo pioraram sistematicamente. A promoção de políticas fiscais expansionistas em um cenário de nítida deterioração dos resultados fiscais pode ter sido um elemento relevante no processo de piora do estado de confiança, contribuindo para reduzir a eficiência da política fiscal.

O resultado gerado pelo modelo, ao sugerir que a indicação de política econômica deve levar em conta suas relações com o estado de confiança, contrapõem-se à receita de bolo defendida por parte substantiva das análises keynesianas. Não necessariamente a realização de uma política fiscal expansionista será eficiente para alavancar o produto. A resposta mecânica (quase automática) às flutuações da atividade deixa de fazer sentido, recuperando-se, assim, a visão da economia como um sistema complexo e no qual não existem respostas pré-definidas. Finalmente, é importante ressaltar que os resultados encontrados no modelo não sugerem que a política fiscal é necessariamente ineficiente; apenas que sua eficiência depende das condições fiscais vigentes e das relações existentes entre o estado de confiança e a política econômica.

\section{Referências}

BLANCHARD, O. J.; FISCHER, S. Lectures on macroeconomics. Cambridge, MA: The MIT Press, 1989.

CARVALHO, F. C. O retorno de Keynes. Nouos Estudos Cebrap, São Paulo, n. 83, p. 91-101, mar. 2009.

DAVIDSON, P. Financial markets, money and the real world. Cheltenham: Edward Elgar Publishing Limited, 2002.

. Money and the real world. London: The MacMillan Press, 1972.

FRITZ, B.; PAULA, L. F; PRATES, D. M. Hierarquia de moedas e redução da autonomia de política econômica em economias periféricas emergentes: uma análise keynesianoestruturalista. In: FERRARI FILHO, F.; TERRA, F. H. B (Org.). Keynes: ensaios sobre os 80 anos da Teoria Geral. Porto Alegre: Tomo Editorial, 2016. p. 288

HARVEY, J. T. Modeling financial crises: a schematic approach. Journal of Post Keynesian Economics, v. 33, n. 1, p. 61-81, 2010.

HICKS, J. R. Mr. Keynes and the "classics": a suggested interpretation. Econometrica, v. 5, n. 2, p. 147-159, Apr., 1937.

KEYNES, J. M. A treatise on money: the pure theory of money and the applied theory of money. Eastford: Martino Fine Books, 2011.

. A treatise on probability. London: Macmillan and Co. Limited, 1921. 
The general theory of employment, interest and money. New York: Harcourt, Brace and Company, 1936.

KINDLEBERGER, C. P. Manias, panics and crashes: a history of financial crises. Hoboken: John Wiley and Sons, 2000.

MEADE, J. E. A simplified model of Mr. Keynes' system. The Review of Economic Studies, v. 4, n. 2, p. 98-107, Feb. 1937.

MINSKY, H. P. Can "it" happen again? Essays on instability and finance. New York: M.E. Sharpe Inc., 1982.

. Stabilizing an unstable economy. London: Yale University Press, 1986.

OREIRO, J. L. Bolhas racionais, ciclos de preços de ativos e racionalidade limitada: uma avaliação crítica dos modelos neoclássicos de bolhas especulativas. Revista Análise Econômica, Porto Alegre, v. 21, n. 40, p. 89-114, set. 2003.

. Capital mobility, real exchange rate appreciation, and asset price bubbles in emerging economies: a Post Keynesian macroeconomic model for a small open economy. Journal of Post Keynesian Economics, v. 28, n. 2, p. 317-344, 2005.

PAULA, L. F. Sistema financeiro, bancos e financiamento da economia: uma abordagem keynesiana. São Paulo: Editora Campus, 2014.

SARGENT, T. J. Macroeconomic theory. 2. ed. San Diego: Academic Press Inc., 1987.

TAYLOR, L.; O'CONNEL, S. A. A Minsky crisis. The Quarterly Journal of Economics, v. 100, Supplement, p. 871-885, 1985.

TOBIN, J. A general equilibrium approach to monetary theory. Journal of Money, Credit and Banking, v. 1, n. 1, p. 15-29, 1969.

Recebido em: 03/04/2017. Aceito em: 04/05/2017. 